\title{
Atomistic study of SiN based ReRAM with high program/ erase cycle endurance
}

\author{
Keita Yamaguchi' ${ }^{a}$, Hiroki Shirakawa, and Kenji Shiraishi \\ Graduate School of Engineering, Nagoya University, \\ Furo-cho, Chikusa-ku, Nagoya 464-8601, Japan \\ a)yamaguchi.keita@b.mbox.nagoya-u.ac.jp
}

\begin{abstract}
We used ab initio calculations to clarify the atomistic origin of memory state switching in SiN based ReRAMs. The results of our calculations indicate that a $\mathrm{N}$ vacancy with a $\mathrm{H}$ atom acts as the high resistance state (HRS) and a $\mathrm{N}$ vacancy without a $\mathrm{H}$ atom is low resistive state (LRS). Moreover, we find that HRS and LRS can be switched by charge injection and removal. These results indicate that having $\mathrm{N}$ vacancies in the SiN layer can enable SiN based ReRAMs with high program/erace cycle endurance.
\end{abstract}

Keywords: first principles calculation, ReRAM, SiN

Classification: Electron devices, circuits and modules

\section{References}

[1] X. Jiang, et al.: "a-SiNx: H-based ultra-low power resistive random access memory with tunable Si dangling bond conduction paths," Sci. Rep. 5 (2015) 15762 (DOI: 10.1038/srep15762).

[2] S. Kim, et al:: "Fully Si compatible SiN resistive switching memory with large self-rectification ratio," AIP Adv. 6 (2016) 015021 (DOI: 10.1063/1.4941364).

[3] A. Mehonic, et al:: "Electrically tailored resistance switching in silicon oxide," Nanotechnology 23 (2012) 455201 (DOI: 10.1088/0957-4484/23/45/455201).

[4] J. P. Perdew, et al.: "Generalized gradient approximation made simple," Phys. Rev. Lett. 77 (1996) 3865 (DOI: 10.1103/PhysRevLett.77.3865).

[5] P. E. Blöchl: "Projector augmented-wave method," Phys. Rev. B 50 (1994) 17953 (DOI: 10.1103/PhysRevB.50.17953).

[6] G. Kresse and J. Hafner: "Ab initio molecular dynamics for liquid metals," Phys. Rev. B 47 (1993) 558 (DOI: 10.1103/PhysRevB.47.558).

[7] G. Kresse and J. Hafner: "Ab initio molecular-dynamics simulation of the liquid-metal-amorphous-semiconductor transition in germanium," Phys. Rev. B 49 (1994) 14251 (DOI: 10.1103/PhysRevB.49.14251).

[8] G. Kresse and C. J. Furthmüller: "Efficiency of ab-initio total energy calculations for metals and semiconductors using a plane-wave basis set," Comput. Mater. Sci. 6 (1996) 15 (DOI: 10.1016/0927-0256(96)00008-0).

[9] G. Kresse and J. Furthmüller: "Efficient iterative schemes for ab initio totalenergy calculations using a plane-wave basis set," Phys. Rev. B 54 (1996) 11169 (DOI: 10.1103/PhysRevB.54.11169).

[10] J. Schneider, et al:: "Structure refinements of $\beta$-Si3N4 at temperatures up to $1360^{\circ} \mathrm{C}$ by X-Ray powder investigation," Z. Kristallogr. 209 (1994) 328 (DOI: 10.1524/zkri.1994.209.4.328). 
crystal, volumetric and morphology data," J. Appl. Cryst. 44 (2011) 1272 (DOI: 10.1107/S0021889811038970).

[12] K. Yamguchi, et al:: "Atomistic design of guiding principles for high quality metal-oxide-nitride-oxide-semiconductor memories: First principles study of $\mathrm{H}$ and $\mathrm{O}$ incorporation effects for $\mathrm{N}$ vacancies in SiN charge trap layers," J. Appl. Phys. 50 (2011) 04DD05 (DOI: 10.7567/JJAP.50.04DD05).

[13] J. Robertson: "Band offsets of wide-band-gap oxides and implications for future electronic devices," J. Vac. Sci. Technol. B 18 (2000) 1785 (DOI: 10. 1116/1.591472).

[14] M. Houssa, et al.: "Trap-assisted tunneling in high permittivity gate dielectric stacks," J. Appl. Phys. 87 (2000) 8615 (DOI: 10.1063/1.373587).

[15] S. Yu, et al.: "Conduction mechanism of TiN/HfOx/Pt resistive switching memory: A trap-assisted-tunneling model,” Appl. Phys. Lett. 99 (2011) 063507 (DOI: 10.1063/1.3624472).

\section{Introduction}

Resistance switching memories (ReRAM) are expected to become major devices in the next generation of nonvolatile memories. This type of memory can provide high speed, low-current operation and back-end-of-line integration [1]. In order to be compatible with conventional complementary metal-oxide-semiconductor (CMOS) technology, it is desirable for ReRAMs to be based on Si. One promising type of Si-based ReRAM reported has a SiN memory storage layer [1, 2]. Other Si-based types of ReRAM using $\mathrm{SiO}_{2}$ have also been reported [3], but SiN-based ones have the benefit of being able to be driven at low voltages [2].

The memory state of a ReRAM is determined by its resistance, which is switched by the bias voltage applied to the memory storage layer. In SiN-based ReRAMs, the cause of the change in resistivity is the termination by $\mathrm{H}$ atoms of $\mathrm{Si}$ dangling bonds in the SiN layer (Fig. 1) [1]. By removing and installing $\mathrm{H}$ atoms, we can control the density of the dangling bonds. As a result, the resistivity of the $\mathrm{SiN}$ layer is switched by constructing or preventing a leakage path through the $\mathrm{Si}$ dangling bonds. In previous papers, it has been reported that ReRAMs with a $\mathrm{Si}_{3} \mathrm{~N}_{4}$ like data storage layer have low leakage current and high program/erase (P/E) cycle endurance compared to ones with Si-rich SiN [1]. However, the atomistic origin of such memory characteristics has not yet been clarified. In this study, we used first principles calculations to investigate the effect of $\mathrm{P} / \mathrm{E}$ operations on the behavior of the Si dangling bonds and the $\mathrm{Si}-\mathrm{H}$ bonds in the $\mathrm{SiN}$ layer.

Our results revealed that $\mathrm{Si}-\mathrm{H}$ bonds at $\mathrm{N}$ vacancies in $\mathrm{Si}_{3} \mathrm{~N}_{4}$ can be removed and recovered by changing the bias voltage. The $\mathrm{P} / \mathrm{E}$ cycles cause this type of defect to behave reversibly. In addition, $\mathrm{N}$ vacancies without $\mathrm{H}$ atoms produce a defect level near the bandgap of bulk Si that behaves as a conduction path. On the other hand, this defect level shifts away from the bandgap of bulk Si when the dangling bonds are terminated by $\mathrm{H}$ atoms. Thus, it was found that these defect states correspond to the high resistance state (HRS) and the low resistance state (LRS) of the ReRAM. These results indicate that the P/E cycle endurance of SiN based ReRAMs can be improved by increasing the number of $\mathrm{N}$ vacancies in the SiN layer. 


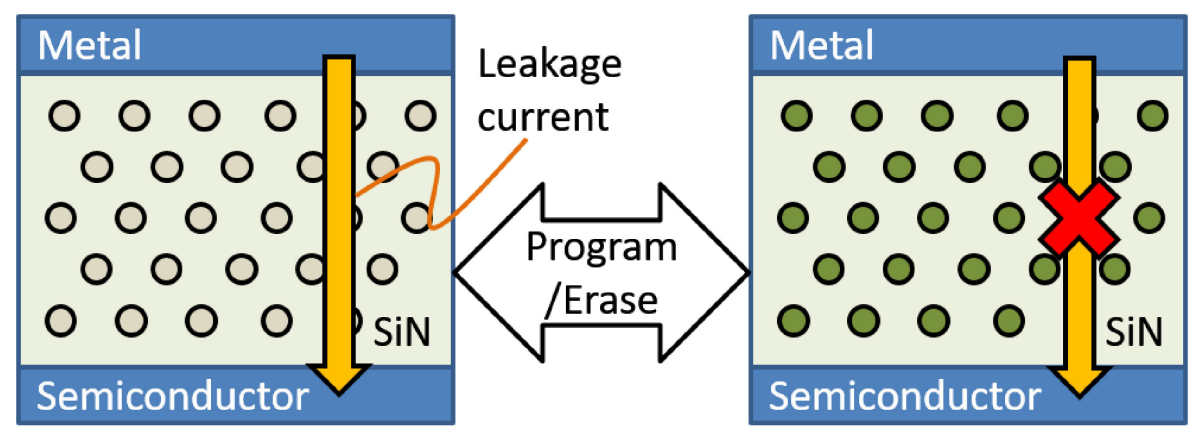

\section{$\mathrm{O}:$ Si dangling bond \\ $\mathrm{O}: \mathrm{Si}-\mathrm{H}$ termination}

Fig. 1. Schematic illustration of $\mathrm{SiN}$ based ReRAM.

\section{Calculation method and conditions}

The models for the calculations were constructed to investigate the behavior of defects in the $\mathrm{Si}_{3} \mathrm{~N}_{4}$ like $\mathrm{SiN}$ layer, which is the ReRAM's data storage layer. We used a 168 -atom supercell of $\beta$ - $\mathrm{Si}_{3} \mathrm{~N}_{4}$ (Fig. 2) for the bulk SiN layer. In addition, in this supercell we introduced one $\mathrm{N}$ vacancy defect to enable a Si dangling bond or $\mathrm{Si}-\mathrm{H}$ bond to be formed.

To discuss the stability of $\mathrm{H}$ termination of the dangling bonds, we introduce the termination energy $\left(E_{\text {Term }}\right)$ of the Si-H bond, which is given by Eq. (1), below:

$$
E_{\text {Term }}(q)=\frac{\left[E_{w_{H}}(q)-E_{w o_{H}}(q)-\frac{n E_{H_{2}}}{2}\right]}{n}
$$

where $E_{w_{H}}(q)$ and $E_{w O_{H}}(q)$ are the total energies of the defect models with charge state $q$ containing and not containing $\mathrm{H}$ atoms, respectively. $n$ is the number of $\mathrm{H}$ atoms in the defect model. The charged defect was simulated by injecting holes/electrons into the supercell with the $\mathrm{N}$ vacancy defect. We assumed that the $\mathrm{H}$ atoms move in the SiN layer during the $\mathrm{P} / \mathrm{E}$ operations. $E_{\mathrm{H}_{2}}$ is the total energy of an $\mathrm{H}_{2}$ molecule in the SiN layer, and is given by Eq. (2).

$$
E_{H 2}=E(b u l k+H 2)-E(b u l k),
$$

where $E($ bulk $)$ and $E($ bulk $+H 2)$ are the total energies of the bulk $\mathrm{Si}_{3} \mathrm{~N}_{4}$ supercell and a supercell with an $\mathrm{H}_{2}$ molecule in the neutral charge state. Fig. 3 shows the stable structure of this $\mathrm{H}_{2}$ molecule model. Here, the molecule is in a six-membered ring composed of $\mathrm{Si}$ and $\mathrm{N}$ atoms.

$\mathrm{Ab}$ initio calculations were performed in the framework of density functional theory (DFT) with the Perdew-Burke-Enzerhof generalized gradient approximation (GGA) [4] and the projected-augmented-wave potential [5] as implemented in the VASP (Vienna ab initio simulation package) code $[6,7,8,9]$. The cutoff energy of the plane-wave basis set was $500 \mathrm{eV}$. $k$ points were sampled with a $2 \times 2 \times 3$ Monkhorst-Pack grid for Brillouin zone integration. All the atoms were relaxed until the atomic force became smaller than $10 \mathrm{meV} / \AA$. We also confirmed that the spin-polarized effect is negligibly small in our calculations. The calculated bulk- $\beta-\mathrm{Si}_{3} \mathrm{~N}_{4}$ properties are in very good agreement with the experimental results. The lattice constants, $\boldsymbol{a}$ and $\boldsymbol{c}$, obtained were $7.66 \AA$ and $2.93 \AA$, respectively, which 
agree well with the experimental values, $\boldsymbol{a}=7.61 \AA$ [10] and $\boldsymbol{c}=2.91 \AA$ [10]. We used VESTA (visualization for electronic and structural analysis) to draw the structures from the results of our calculations [11].

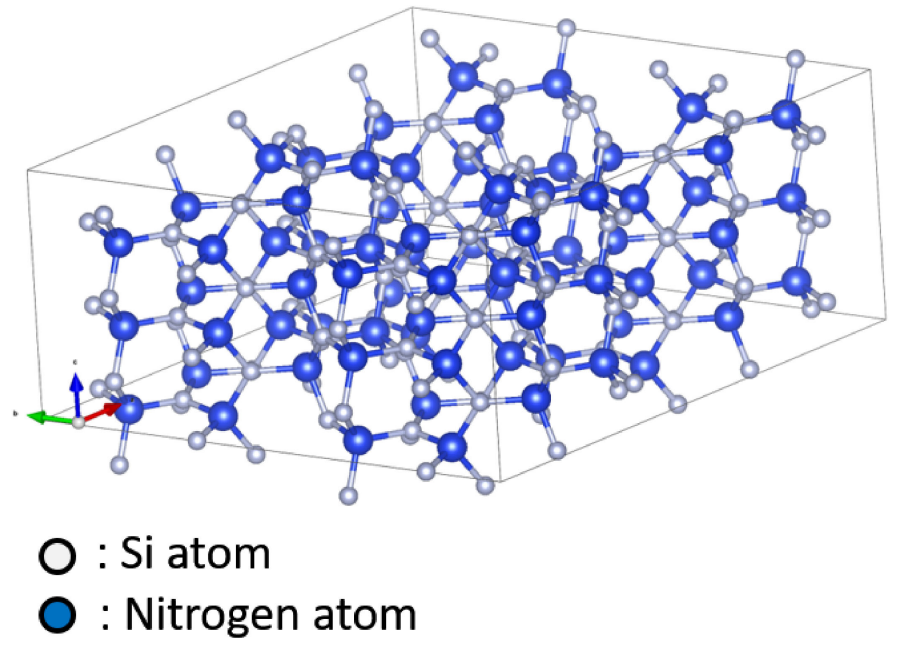

Fig. 2. A 168-atom supercell of $\beta-\mathrm{Si}_{3} \mathrm{~N}_{4}$ used to model the $\mathrm{SiN}$ memory layer.

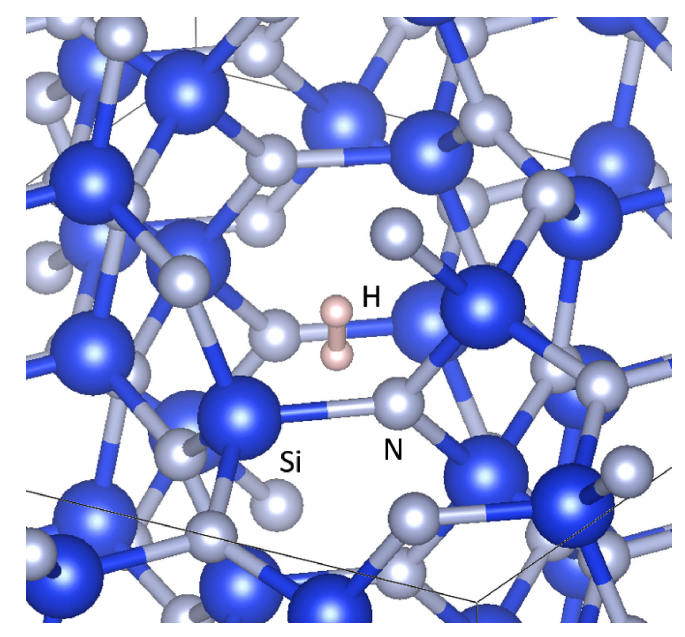

Fig. 3. Stable structure with an $\mathrm{H}_{2}$ molecule in the $\beta-\mathrm{Si}_{3} \mathrm{~N}_{4}$ supercell.

\section{Results and discussions}

First, we show the characteristics of the $\mathrm{N}$ vacancy defect model with and without an $\mathrm{H}$ atom. The stable atomistic structure of the model with an $\mathrm{N}$ vacancy in the neutral charge state is shown in Fig. 4(a). Here, the three Si atoms around the N vacancy make an isosceles triangle, and all the $\mathrm{N}$ atoms are four-fold coordinated. On the other hand, Fig. 4(b) shows the stable structure of the model with an N vacancy and an $\mathrm{H}$ atom in the neutral charge state. As shown, the three Si dangling bonds are stabilized by forming $\mathrm{Si}-\mathrm{H}$ and $\mathrm{Si}-\mathrm{Si}$ bonds. In this structure, all the $\mathrm{Si}$ atoms are four-fold coordinated and all the $\mathrm{N}$ atoms are three-fold coordinated. This bond network comprises atoms having the ideal coordination number. In our previous paper, an $\mathrm{N}$ vacancy with multiple $\mathrm{H}$ atoms was found to be unstable due to steric hindrance [12]. 


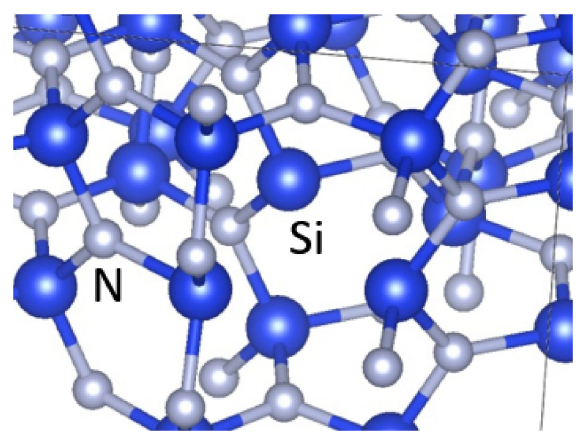

(a)
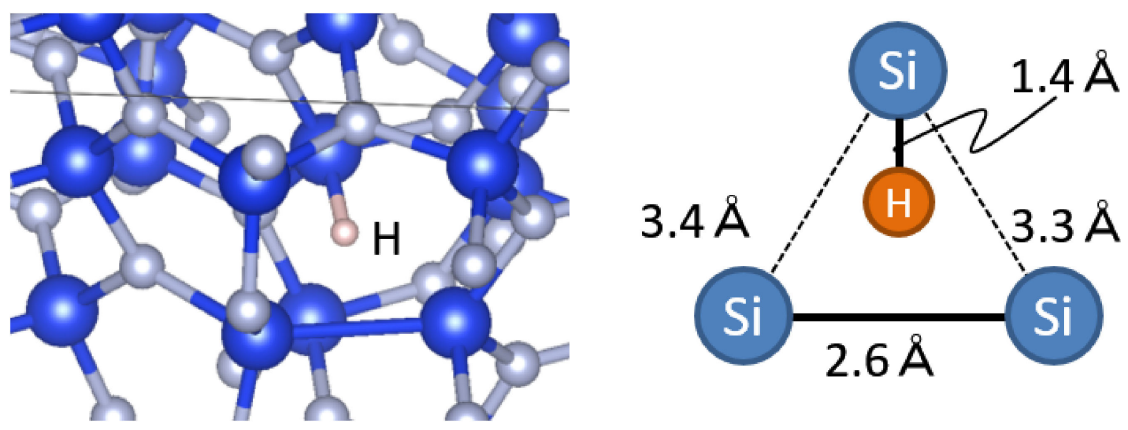

(b)

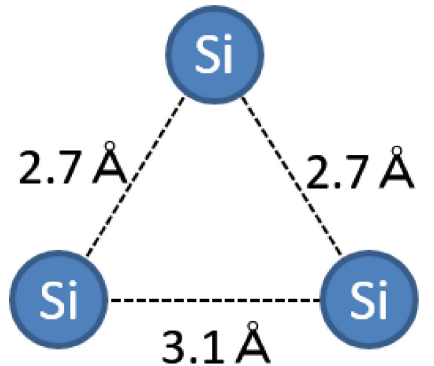

$3.1 \AA$

Fig. 4. (a) The stable atomistic structure of the model with an $\mathrm{N}$ vacancy in the neutral charge state. (b) The stable structure of the model with an $\mathrm{N}$ vacancy and one $\mathrm{H}$ atom in the neutral charge state.

Next, we examine the behavior of this defect during $\mathrm{P} / \mathrm{E}$ operations. The termination energies in the positive $(q=+1)$, negative $(q=-1)$ and neutral charge states are calculated by simulation with negative and positive bias. In the neutral charge state, the termination energy is $-0.76 \mathrm{eV}$. On the other hand, in the positive $(q=+1)$ and negative $(q=-1)$ charge states, the termination energies are 0.43 and $0.70 \mathrm{eV}$, respectively.

These results indicate that the stability of the $\mathrm{H}$ atom in this defect is changed by hole/electron injection. In the neutral charge state, the $\mathrm{H}$ atom in the $\mathrm{N}$ vacancy is stable. However, this $\mathrm{H}$ atom becomes unstable in the positive and negative charge states. Therefore, this defect can reversibly capture and release the $\mathrm{H}$ atom by hole or electron injection due to the $\mathrm{P} / \mathrm{E}$ operation.

Fig. 5(a) and (b) are atomistic structures of the positive charge $(q=+1)$ and negative charge $(\mathrm{q}=-1)$ states of the $\mathrm{N}$ vacancy defect without the $\mathrm{H}$ atom, respectively. In the positive charge state, three $\mathrm{Si}$ atoms around the $\mathrm{N}$ vacancy make an equilateral triangle. On the other hand, in the negative charge state, the three $\mathrm{Si}$ atoms form an isosceles triangle, slightly shorter compared to the one in the neutral charged state. In our previous study, we clarified that these structural and symmetrical changes are caused by the Jahn-Teller effect [12].

To clarify the electrical properties, we calculated the density of states (DOS) of these stable charge states. The DOS and simplified atomistic structures in the stable states are summarized in Fig. 6. The bandgap for bulk $\mathrm{Si}$ is shown in the DOS plots [13]. In the positive and negative charge states, the defect levels are in the middle of 


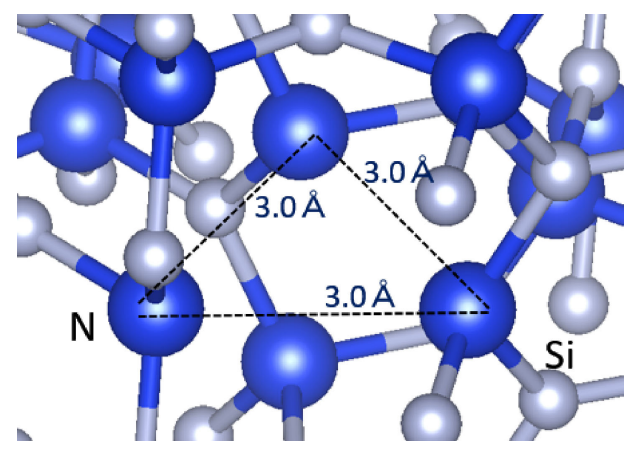

(a)

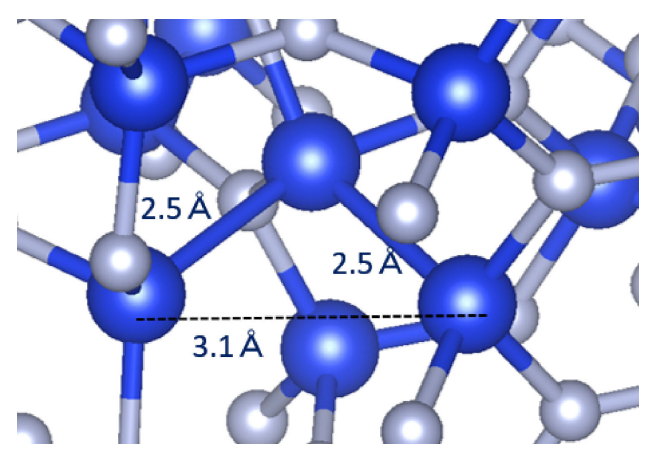

(b)

Fig. 5. (a) The atomistic structures of the positive charge $(q=+1)$ and (b) negative charge $(q=-1)$ states of the $\mathrm{N}$ vacancy defect without the $\mathrm{H}$ atom.
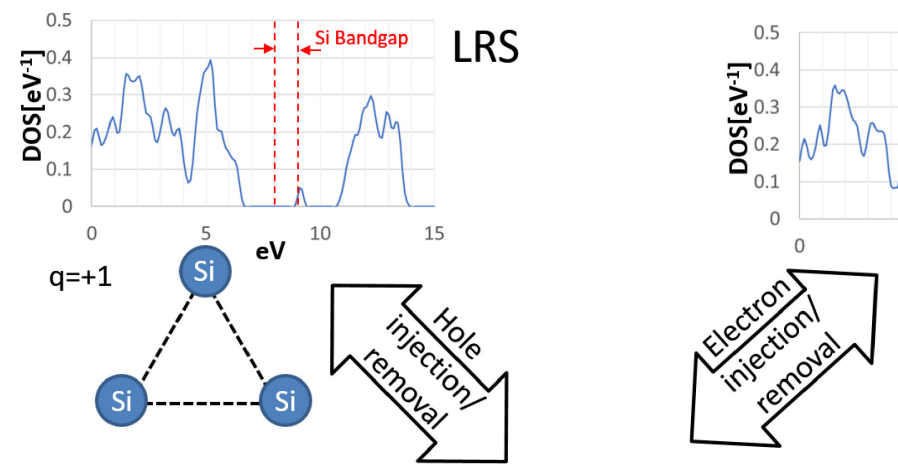

LRS
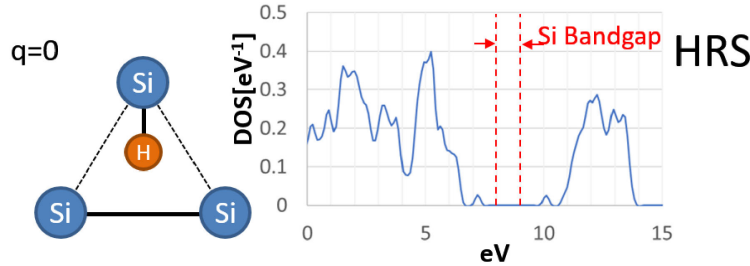

Fig. 6. The DOS and simplified atomistic structures in the $\mathrm{P} / \mathrm{E}$ states.

the $\mathrm{Si}_{3} \mathrm{~N}_{4}$ bandgap. This is in the vicinity of the bandgap of $\mathrm{Si}$, which is the substrate used for SiN based ReRAMs. It is well known that defect levels in this region behave as conduction paths $[14,15]$. Therefore, these charge states correspond to the LRS. On the other hand, in the neutral state, with an $\mathrm{N}$ vacancy and an $\mathrm{H}$ atom, the defect levels shift to near the top of the valence band and the bottom of the conduction band. This state corresponds to the HRS.

These results indicate that this $\mathrm{N}$ vacancy defect in SiN can reversibly change between the HRS and the LRS depending on the bias voltage applied during the $\mathrm{P} / \mathrm{E}$ operations. Therefore, these defects are ideal memory centers in SiN-based ReRAMs. By increasing this type of defect in $\mathrm{SiN}$, it is possible to fabricate a memory having high $\mathrm{P} / \mathrm{E}$ cycle endurance.

\section{Conclusions}

In this study, we used first principles calculations to examine SiN based ReRAMs with high $\mathrm{P} / \mathrm{E}$ cycle endurance. In such memories, the $\mathrm{P} / \mathrm{E}$ operation is realized by 
a positive or negative bias to the memory storage layer, leading to the removal or capture of $\mathrm{H}$ atoms in the SiN layer.

The results of our calculations show that $\mathrm{Si}$ dangling bonds arising due to $\mathrm{N}$ vacancies can capture and release $\mathrm{H}$ atoms during the $\mathrm{P} / \mathrm{E}$ cycles. These dangling bonds cause energy levels located near the Si bandgap that give rise to a conduction path. This state is the origin of the LRS. On the other hand, when the dangling bonds are terminated by $\mathrm{H}$ atoms, these energy levels shift away from the $\mathrm{Si}$ bandgap. This state is the origin of the HRS, which cannot be formed in the model without an $\mathrm{H}$ atom. Therefore, this defect can be switched reversibly between the HRS and the LRS by charge injection and removal. We can improve the P/E cycle endurance of SiN based ReRAMs by increasing the number of this type of defect. 